\title{
Microenvironmental Stress as the Driver of Cell Plasticity and Tumour Autonomy
}

\author{
Dan V Nicolau ${ }^{1,2}$ and Sunil R Lakhani ${ }^{3}$ \\ ${ }^{1}$ School of Mathematical Sciences, Queensland University of Technology, Australia. \\ ${ }^{2}$ Nuffield Department of Experimental Medicine, University of Oxford, Australia \\ ${ }^{3}$ University of Queensland Centre for Clinical Research and Pathology Queensland, Royal Brisbane Women's Hospital, Australia.
}

*Corresponding author: Dan V Nicolau, School of Mathematical Sciences, Queensland University of Technology, Australia

\begin{abstract}
It is well established that the tumour microenvironment (TME) plays a key role in cancer progression, from local invasion all the way to metastasis. In particular, the tumour cell is subjected to various challenges within the TME, from hypoxia to attack by immune cells, collectively rendering its local habitat hostile. The resulting selection pressure essentially encourages invasive behavior by favouring plastic phenotypes. Epigenetic changes then appear to confer on the tumour cell enhanced motility, reduced cell-cell adhesion, the epithelial-mesenchymal transition and other features that have been linked with unicellularity, particularly in the context of the atavistic model of cancer. While some of these changes do indeed appear to be 'regressive' and connected to ancient genes, the atavistic model fails to explain why many others are 'innovative', recapitulating - in pathological form programmes requiring exquisite, multicellular cooperation, including angiogenesis and immune evasion. Taking these aspects of cancer progression together suggests a model whereby the hostile TME selects for autonomy, rather than unicellularity, and hints at a deep duality between cellular autonomy and a failure of support for the selective advantages usually conferred by existence within a coordinated, multicellular community.
\end{abstract}

Keywords: Cancer Progression; Tumour Microenvironment; Plasticity; Atavistic Model

\section{Introduction}

Early models of cancer postulated that somatic mutations in cancer cells were responsible for cancer cell progression Fearon, et al. [1]. We now know that such genetic changes in the tumor cells cannot on their own account for the rapid tumour progression from local invasion to metastasis seen in cancer Taddei, et al. [2]. Instead, it is becoming clear that epigenetic changes, representing adaptations of the abnormal cells to unfavourable environments and the intervention of the immune system contribute substantially to - and are possibly the main driver of - the metastatic cascade. Both environmental factors and cell-cell interactions are involved in these epigenetic alterations, the final outcomes being invasion of the local stroma, access to and survival in lymph and blood, extravasation and colonization of distal sites and, ultimately, resistance to the interventions of the oncologist Lujambia, et al. [3]. However, while there is a huge body of literature focused on understanding the genetic and epigenetic changes that enable and drive cancer progression, existing models are highly diverse and focused on individual pieces of the puzzle. It has continued to be a mystery, for instance, how tumour cells, having undergone a relatively small number of changes relative to normal cells, suddenly develop myriad abilities to evade the actions of the host, as well as to survive, invade, proliferate Davies, et al. [4] grow their own vasculature and so on. In the last decade, a new paradigm has ambitiously attempted to explain the progression of cancer by proposing that cancer represents a reversion to a unicellular state. The so-called atavistic model argues that cancer's origins can be found in the transition from unicellular to multicellular life that occurred soon after the emergence of animals some 600 million years ago Bussey, et al. [5]. Here, we briefly review some of evidence for how adverse conditions within the TME cause epigenetic changes enabling survival. Broadly, these, particularly the epithelial-mesenchymal transition (EMT) are those associated 
with cell plasticity and promote escape from the TME and migration to more favourable environments. In the context of the atavistic model of cancer, these have been proposed to represent activation of ancient, 'unicellular' programmes. A model of 'reversion' of this type, however sophisticated, does not explain the myriad cooperative and sophisticated innovations displayed by tumours, suggesting instead that the hostile TME selects for long-term, cooperative autonomy rather than short-term unicellularity

\section{Cellular Stress-Induced Adaptive Tumour Cell Plasticity}

It is by now well established that the aforementioned adverse conditions within the TME cause insults to tumour cells, leading to adaptive responses, allowing the cell to survive and, respectively, leave its hostile microenvironment. These changes are broadly those associated with cell plasticity and include increased cell motility, resistance to apoptosis, metabolic reprogramming and matrix invasion, in part through direct reprogramming of the ECM Taddei, et al. [2]. In concert, they appear to confer to the tumour cell three types of selective advantage: (1) enhanced survival within the adverse microenvironment and (2) the ability to escape its hostile site and migrate to more favourable environments and (3) the ability to coerce normal, local cells to aid in the growth of the tumour colony. It is on the second aspect of tumour cell plasticity that we focus here: the capacity to escape the toxic local environment. One of the primary motifs in cancer cell plasticity-still not well understoodis the epithelial-mesenchymal transition (EMT) Dongre, et al. [6]. EMT is a reversible process, comprising a regulated, epigenetic programme involving the reorganisation of the cytoskeleton and loss of normal cell-cell and cell-matrix cooperative interactions, leading, ultimately, to either the loss of the cell's epithelial features as well as with expression of mesenchymal features and markers Jing, et al. [8]. An increasingly large number of transcription factors have by now been implicated in this programme Sun, et al. [7]. Of interest here, diverse lines of evidence strongly suggest that ECM is triggered by inflammatory stress within the tumour microenvironment, caused by hypoxia, the action of immune cells and other factors Jing, et al. [8]. Remarkably, EMT increasingly appears to be a transient sine qua non for progression in many solid cancers, apparently by imparting on the cell adaptive traits including enhanced motility, increased ability to disseminate Dongre, et al. [6] and resistance to therapeutics Singh, et al. [9]; Shibue, et al. [10] In addition, the completion of EMT correlates to an increase in cells apoptosis resistance, allowing cells to survive as they progress from the TME to local and distal invasion Taddei, et al. [2]. A major feature of EMT-led cell plasticity, as already mentioned, is the loss of normal cell-cell and cell-matrix interactions. Specifically, it appears that the downregulation of expression of genes in the E-cadherin family, mediated by several transcription factors Canesin, et al. [11] is a hallmark of EMT. Through the loss of E-cadherin during EMT, polarised epithelial cells undergo substantial transcriptional and morphological changes, resulting in loss of cell-cell adhesion Elisha, et al. [12], setting the stage for the altered cells' ability to migrate away from the tumour microenvironment, an indispensable step in the invasion sequence. Specifically, as the EMT programme advances, the cells lose or loosen their junctional connections, as well as detaching from the epithelial layer whence they originated. This modified phenotype is characterised by enhanced migratory activity and invasiveness Kaluri, et al. [13]; Yilmaz et al. [14]; Thiery et al. [15]. Since it is now thought that local cellular stress within the tumour microenvironment directly induces the EMT Jing, et al. [8], it is clear that there is a direct line from the emergence of this local environmental hostility during microtumour growth and the loss of cell-cell adhesion, establishing the cellular autonomy necessary for migration away from the hostile site. As mentioned above, the EMT, in addition to its role in the loss off cell-cell adhesion and acquisition of cellular autonomy, is itself directly also associated with enhanced motility of the EMT-modified cells Lamouille, et al. [16]. The molecular changes involved, including (a) reorganisation of the cortical actin cytoskeleton into one enabling dynamic cell elongation and directional motility and (b) actin-rich membrane projections facilitating cell movement, are fairly well described Yilmaz, et al. [17] Ridley [18]. The resulting increase in cancer cell motility is dramatic: for instance, it has been known for nearly two decades that carcinoma cells in the primary tumour can move at up to 10 times the velocity of similar cells in vitro and, specifically, that the highest velocities are observed in carcinoma cells showing evidence of having undergone EMT Condeelis, et al. [19].

However, separately from the EMT, we now also know that cancer cells exhibit a switch from mesenchymal to amoeboid motility (MAT), which confers additional autonomy and mobility to the cell Taddei et al. [2]. Ameboid motility — a squeezing movement of the cell, not dependent on metalloproteases or integrin leakage - appears to allow it to cross matrix barriers as well as to resist therapeutics that target proteases and integrins, respectively. This transition also seems to be induced by local cellular stress attributable to hypoxic tumour environments, specifically associated with the release of reactive oxygen and nitrogen species Friedl [20] Yilmaz et al. [17] Pani et al. [21], a view consistent with increasingly sophisticated models of cell motility modes Friedl, et al. [22]. Myriad other factors contribute to the development of tumour cell plasticity within the TME. These include stiffness of the extracellular matrix (ECM) Taddei, et al. [2], in which increasing ECM fibre density traps the cancer cell Brabek et al. [23], promoting an active adaptation of cancer cells whereby their protrusive forces are increased in order to compensate for the higher hinderance of the matrix Wolf et al. [24]. Cancer-associated macrophages (CAMs) and other immune cells produce stress on tumour cells, further promoting plasticity, including by initiation of the EMT Wu et al. [25]. Finally, EMT stress leads to resistance to apoptosis in tumour cells Yaacoub et al. [26]. The unifying theme is, as above, that of cellular stress leading to epigenetic changes conferring plasticity on the tumour cell. 


\section{The Atavistic Model Of Cancer}

Several years ago, a novel view of cancer proposed that progression is attributable to a 'reversion' of cancer cells to a unicellular state Pennisi [27]. This so-called "atavistic model" of cancer Davies et al. [30] begins with the observation that many of the genes associated with unicellular life seem to be involved in cancer states and goes on to propose that genetic or epigenetic malfunctions in cancer unlock an ancient 'toolkit' of pre-existing adaptations associated with loose-knit, only partially differentiated colonies of early animals. Indeed, at a much higher level than that of the genome, the idea that cancer recapitulates the phenotypes of ancient animals was first suggested nearly a century ago Boveri [28]. More specifically, the atavistic theory postulates that the origins of cancer can be found in the transitional phase from unicellular to multicellular life, perhaps in the early stages following the emergence of metazoans (animals) circa 600 million years ago Bussey et al. [5]. Fascinatingly, the atavistic model would seem to hint at a resolution to a long-standing mystery in cancer: how cancers appear to suddenly and all at once deploy a wide array of survival and evasive strategies against the host Davies et al. [30]. These 'tricks' include silencing of tumour suppressor genes, switching off senescence, apoptosis and anoikis, and evading the immune system Butcher et al. [29]. In the atavistic view, cancer cells are the heirs of unicellular or early multicellular life and carry in their genomes an ancient survival toolkit which, once "opened", confers these sophisticated survival abilities to the cells "at once". In addition, the atavistic model is theoretically able to suggest, a priori, novel therapeutic approaches, since the genes associated with unicellularity would also be more likely to be those involved in cancer progression Linewater et al. [30]. Recent evidence lends support to at least some aspects of the atavistic model. Analysis of the functional differences between cell types at the gene expression level for 107 different cell types and 18 tumour types revealed a consistent progression of all tumour types towards a similar stem-like state, which appears to be the result of selection for unicellular states (rather than pluripotency) through the loss of features of multicellularity and positive selection for "primitive functions" Chen et al. [31]. A study incorporating phylogenetic and interaction data into expression analysis of seven solid tumours revealed universal strong preferential expression of genes shared with unicellular species Trigos et al. [32] as well as disruption of links between unicellular and multicellular components of gene regulatory networks. Furthermore, there appears to be a small "interface" between the genes associated with multi- and unicellularity in these cancers, suggesting that disruption of cross-talk between older and younger genes may prove to be a key factor in the onset of tumour genesis Trigos et al. [32]; Bussey et al. [5].

\section{Towards Integrative Models of Tumour Cell Invasion}

Taken together, these and other Domazet-Lošo et al. [34] lines of enquiry point towards support for a model in which cancer - particularly cancer progression, from local invasion to metastasis - represents at least to some extent a failure of support for highly coordinated, multicellular behaviour. In this context, the emergence of multicellularity 600 million years ago can be seen as having "set the stage" for the emergence of cancer Trigos et al. [32]. Consistent with this view, we now know, for example, that cancers occur in very ancient metazoans and thus that cancer appears, indeed, to have emerged almost concomitantly with multicellularity Domazet-Lošo et al. [34]. On the other hand, the atavistic model fails to explain a number of phenomena. Strikingly, for instance, even evidence in support of the model fails to explain the existence of stem cells Trigos et al. [32], whereas dedifferentiation to stem cell phenotypes is a hallmark of cancer. Furthermore, the atavistic model suggests that early metazoa would have at least some of the morphological properties of tumours, which is not the case. Perhaps most compellingly, it is clear that innovations such as angiogenesis, immune evasion and tissue infiltration, all features of cancer progression, cannot be explained by a 'reverse' evolution to a unicellular phenotype Chen et al. [35] which, by definition, would be naïve to the existence of blood, immunity and tissue, respectively. Tumour progression as a whole can only be ultimately understood in the broader context of the interaction of the cancer cells not only with other (normal or abnormal) cells but also with the larger environment. Cell migration takes place-first-locally, within the confines of the TME, before penetration outside the basement membrane and access to lymph and blood. At each stage of the process, tumour cells are subjected to cellular stress either from the local environment, the immune response and the action of drugs. It stands to reason that these different kinds of cellular stress would lead to different, context-dependent evolutionary selection pressures, some of which may be met by the activation of ancient, atavistic programmes while others would not.

A unifying feature of these different stresses, however, may be that they disproportionately affect non-autonomous cells that operate in tight cooperation with their local environment. Consequently, cellular stress in the TME as well as outside it would select for cell populations able to survive through the activation of autonomous genetic programmes (some of which may indeed be ancient). Therefore, it is plausible that autonomy, rather than atavism, is the motif that unifies tumour cell plasticity, tumour heterogeneity, cellular stress states and cancer progression more generally, as illustrated in (Figure 1). In addition to explaining innovative features of invasion, such a model also naturally accounts for stem cells, the EMT and 'reversions' to primitive motility modes. This autonomy need not be expressed at the level of only the individual cell but ultimately at the level of the in situ lesion and, later, the colonies associated with distal spread. In this context, both experimental and modelling studies targeted at elucidating the degree to which autonomy, appropriately defined, is present in cells at different stages of carcinogenesis, have the potential to shed light on the outlines of the cancer progression process. 


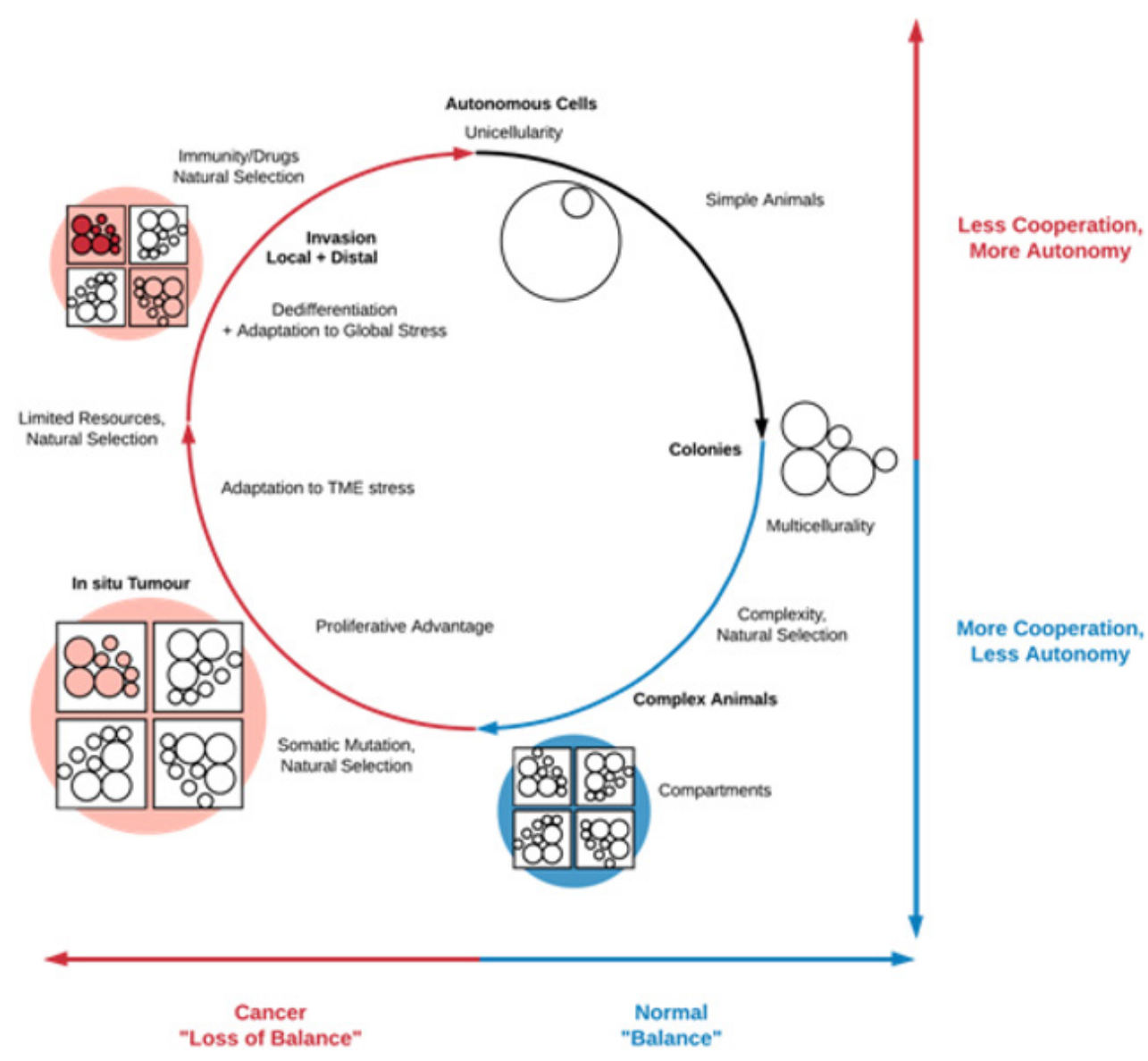

Figure 1: Loss of balance in cancer may be the result of stress-induced evolutionary selection for the activation of autonomous cellular programmes that allow abnormal cells to survive in hostile environments. While the unifying feature of these adaptations would be autonomy, in some cases these would be innovative (e.g. immune system evasion) while in others they would be atavistic (e.g. reversion to primitive motility modes). At the level of the TME, for instance, tumour cell cooperation during local invasion would be an example of a programme exploiting highly multicellular processes but ultimately resulting in individual cell autonomy (post-TME escape), while loss of cell-cell adhesion during the local invasion process - an 'atavistic' adaptation - would more directly confer autonomy on the cell.

\section{References}

1. Fearon Eric R, Bert Vogelstein (1990) A Genetic Model for Colorectal Tumorigenesis. Cell 61(5): 759-767.

2. Taddei Maria Letizia, Elisa Giannoni, Giuseppina Comito, Paola Chiarugi (2013) Microenvironment and Tumor Cell Plasticity: An Easy Way Out. Cancer Letters 341(1): 80-96.

3. Lujambio, Amaia, Scott W Lowe (2012) The Microcosmos of Cancer Nature 482(7385): 347-355.

4. Davies, P C W, C H Lineweaver (2011) Cancer Tumors as Metazoa 1.0: Tapping Genes of Ancient Ancestors. Physical Biology 8(1): 015001.

5. Bussey, Kimberly J, Luis H Cisneros, Charles H Lineweaver, Paul CW Davies (2017) Ancestral Gene Regulatory Networks Drive Cancer. Proceedings of the National Academy of Sciences 114(24): 6160-6162.

6. Dongre Anushka, Robert A Weinberg (2019) New Insights into the Mechanisms of Epithelial-Mesenchymal Transition and Implications for Cancer. Nature Reviews Molecular Cell Biology 20(2): 69-84.

7. Sun Lidong, Jia Fang (2016) Epigenetic Regulation of EpithelialMesenchymal Transition. Cellular and Molecular Life Sciences 73(23): $4493-4515$.
8. Jing Yingying, Zhipeng Han, Shanshan Zhang, Yan Liu, Lixin Wei, et al. (2011)Epithelial-Mesenchymal Transition in Tumor Microenvironment." Cell \& Bioscience 1(1): p. 29.

9. Singh A, Settleman J (2010) EMT, Cancer Stem Cells and Drug Resistance: An Emerging Axis of Evil in the War on Cancer. Oncogene 29(34): 47414751.

10. Shibue Tsukasa, Robert A Weinberg (2017) EMT, CSCs, and Drug Resistance: The Mechanistic Link and Clinical Implications. Nature Reviews Clinical Oncology 14(10): 611-629.

11. Canesin G, E P Cuevas, V Santos, C López-Menéndez, G Moreno-Bueno, et al. (2015) Lysyl Oxidase-like 2 (LOXL2) and E47 EMT Factor: Novel Partners in E-Cadherin Repression and Early Metastasis Colonization. Oncogene 34(8): 951-964.

12. Elisha Yair, Vyacheslav Kalchenko, Yuri Kuznetsov, Benjamin Geiger (2018) Dual Role of E-Cadherin in the Regulation of Invasive Collective Migration of Mammary Carcinoma Cells. Scientific Reports 8(1): 4986.

13. Kalluri Raghu, Robert A Weinberg (2009) the Basics of EpithelialMesenchymal Transition. Journal of Clinical Investigation 119(6): 14201428. 
14. Yilmaz Mahmut, Gerhard Christofori (2009) EMT, the Cytoskeleton, and Cancer Cell Invasion. Cancer and Metastasis Reviews 28(1-2): 15-33.

15. Thiery Jean Paul, Hervé Acloque, Ruby YJ Huang, M. Angela Nieto (2009) Epithelial-Mesenchymal Transitions in Development and Disease Cell 139(5): 871-890

16. Lamouille, Samy, Jian Xu, Rik Derynck Molecular Mechanisms of Epithelial-Mesenchymal Transition. Nature Reviews Molecular Cell Biology 15(3): 178-196.

17. Yilmaz Mahmut, Gerhard Christofori (2010) Mechanisms of Motility in Metastasizing Cells. Molecular Cancer Research 8(5): 629-642.

18. Ridley Anne J (2011) Life at the Leading Edge. Cell 145(7): 1012-1022.

19. Condeelis John, Jeffrey E Segall (2003) Intravital Imaging of Cell Movement in Tumours. Nature Reviews Cancer 3(12): 921-930.

20. Friedl Peter (2004) Prespecification and Plasticity: Shifting Mechanisms of Cell Migration. Current Opinion in Cell Biology 16(1): 14-23.

21. Pani Giovambattista, Elisa Giannoni, Tommaso Galeotti, Paola Chiarugi (2009) Redox-Based Escape Mechanism from Death: The Cancer Lesson." Antioxidants \& Redox Signaling 11(11): 2791-2806.

22. Friedl Peter, Katarina Wolf (2010) Plasticity of Cell Migration: A Multiscale Tuning Model. The Journal of Cell Biology 188(1): 11-19.

23. Brábek, Jan, Claudia T Mierke, Daniel Rösel, Pavel Veselý, Ben Fabry (2010) the Role of the Tissue Microenvironment in the Regulation of Cancer Cell Motility and Invasion." Cell Communication and Signaling: CCS 8: p. 22.

24. Wolf Katarina, Peter Friedl (2011) Extracellular Matrix Determinants of Proteolytic and Non-Proteolytic Cell Migration. Trends in Cell Biology 21(12): 736-744.

25. Wu Yadi, Jiong Deng, Piotr G, Rychahou Suimin Qiu B Mark Evers (2009) Stabilization of Snail by NF-KappaB Is Required for InflammationInduced Cell Migration and Invasion. Cancer Cell 15(5): 416-428.
26. Yaacoub Katherine, Remy Pedeux, Karin Tarte, Thierry Guillaudeux (2016) Role of the Tumor Microenvironment in Regulating Apoptosis and Cancer Progression. Cancer Letters 378(2): 150-59.

27. Pennisi Elizabeth (2018) Is Cancer a Breakdown of Multicellularity?" Science 360(6396): 1391-1391.

28. Boveri T (1929) the Origins of Malignant Tumors. Williams \& Wilkins; Baltimore 44: 506.

29. Butcher Darci T, Tamara Alliston, Valerie M Weaver (2008) A Tense Situation: Forcing Tumour Progression. Nature Reviews Cancer 9(2): 108-122.

30. Lineweaver Charles H, Paul C W Davies, Mark D Vincent(2014) Targeting Cancer's Weaknesses (Not Its Strengths): Therapeutic Strategies Suggested by the Atavistic Model: Insights \& Perspectives BioEssays 36(9): 827-835.

31. Chen Han, Xionglei He (2016) the Convergent Cancer Evolution toward a Single Cellular Destination." Molecular Biology and Evolution 33(1): 4-12.

32. Trigos Anna S, Richard B Pearson, Anthony T Papenfuss, David L Goode (2018) How the Evolution of Multicellularity Set the Stage for Cancer." British Journal of Cancer 118(2): 145-152.

33. Domazet-Lošo, Tomislav, Diethard Tautz (2010) Phylostratigraphic Tracking of Cancer Genes Suggests a Link to the Emergence of Multicellularity in Metazoa. BMC Biology 8(1): p. 66.

34. Domazet-Lošo Tomislav, Alexander Klimovich, Boris Anokhin, Friederike Anton-Erxleben, Mailin J Hamm et al. (2014) Naturally Occurring Tumours in the Basal Metazoan Hydra." Nature Communications 5(1): 4222.

35. Chen Han, Fangqin Lin, Ke Xing, Xionglei He (2015) The Reverse Evolution from Multicellularity to Unicellularity during Carcinogenesis. Nature Communications 6(1): 6367.

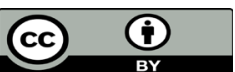

This work is licensed under Creative Commons Attribution 4.0 License

To Submit Your Article Click Here: Submit Article

DOI: $10.32474 /$ OAJOM.2020.03.000173

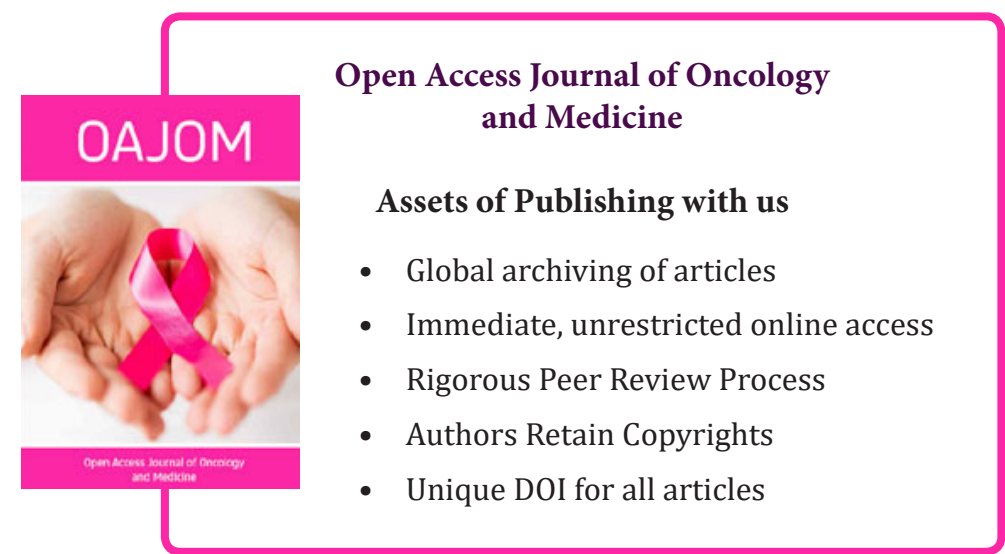

